\title{
Amnésia Induzida por Álcool: prevalência e fatores associados em estudantes de medicina
}

\author{
Alcohol-induced blackouts: prevalence and associated \\ factors in medical students
}

\section{Amnesia inducida por alcohol: prevalencia y factores asociados en estudiantes de medicina}

\author{
Larissa de Souza Mazzuco시 Juliano Della Justina², \\ Eduardo Pacheco Rico ${ }^{3}$
}

\begin{abstract}
1.Acadêmica do curso de Medicina da Universidade do Extremo Sul Catarinense (UNESC), Criciúma-SC, Brasil. Orcid: https://orcid.org/0000-0002-3130-7377

2.Acadêmico do curso de Medicina da Universidade do Extremo Sul Catarinense (UNESC), Criciúma-SC, Brasil. Orcid: https://orcid.org/0000-0001-8207-9469

3.Biólogo, Doutor, Docente do Programa de Pós-graduação em Ciências da Saúde da Universidade do Extremo Sul Catarinense (UNESC), Criciúma-SC, Brasil. Orcid: https://orcid.org/0000-0003-0019-328X
\end{abstract}

\begin{abstract}
Resumo
Objetivo. Conhecer a prevalência e fatores associados à amnésia induzida por álcool (AIA) em acadêmicos de medicina da Universidade do Extremo Sul Catarinense. Método. Pesquisa de caráter observacional transversal, com abordagem quantitativa. Realizada através de um questionário online, elaborado pelos próprios autores, contendo perguntas selecionadas do Alcohol Use Disorder Identification Test (AUDIT). O questionário avaliou a população acadêmica $(n=268)$ do curso de medicina da Universidade do Extremo Sul Catarinense quanto ao seu perfil, consumo de álcool e AIA. Resultados. Foi observado que dos estudantes que bebem 2 a 4 vezes por mês 48,9\% apresentaram AIA, já dos que bebem 4 ou mais vezes por semana $75 \%$ apresentaram AIA. Ao analisar por doses consumidas por ocasião, dos que bebem de 3 a 4 doses 47,6\% apresentaram AIA, e dos que bebem 10 ou mais doses $88 \%$ apresentaram AIA. Quando analisado a frequência de binge drink, dos que bebem menos que mensalmente $45,4 \%$ apresentaram AIA, e semanalmente $67 \%$ apresentaram AIA. Não foi encontrada relação entre sexo, perfil de moradia, semestre da graduação e AIA. Sobre os fatores comportamentais, 59,8\% dos entrevistados participaram de alguma pré-festa ou jogos com bebidas antes da AIA, 54,7\% não se alimentaram corretamente, e 79,5\% relataram ter feito algo durante a AIA que se arrependeu depois. Conclusão. Existe uma alta prevalência de AIA nos estudantes de medicina, sendo esta associada ao consumo de álcool em excesso, ter participado de pré-festas ou jogos com bebidas, não ter se alimentado corretamente e arrependimentos.
\end{abstract}

Unitermos. Consumo de Álcool na Faculdade; Transtornos Relacionados ao Uso de Álcool; Fator de Risco; Amnésia Anterógrada; Estudantes de Medicina

\begin{abstract}
Objective. Evaluate the prevalence and factors associated with alcohol-induced blackouts (AIB) in medical students at the Universidade do Extremo Sul Catarinense. Method. Observational cross-sectional research, with a quantitative approach. Conducted through an online questionnaire, prepared by the authors themselves, and containing selected questions from the from the Alcohol Use Disorder Identification Test (AUDIT). The questionnaire evaluated the academic population $(n=268)$ of the medical graduation course at the extreme south of Santa Catarina regarding their profile, alcohol consumption and alcohol-induced blackouts. Results. It was observed that of the students who drink 2 to 4 times a month, $48.9 \%$ had AIA, whereas of those who drink 4 or more times a week, $75 \%$ had AIB. When analyzing by doses consumed per occasion, of those who drink 3 to 4 doses $47.6 \%$ had AIB, and of those who drink 10 or more doses $88 \%$ had AIB. When analyzing the frequency of binge
\end{abstract}


drink, $45.4 \%$ of those who drink less than monthly had AIB, and $67 \%$ weekly had AIB. No relationship was found between sex, housing profile, graduation semester, and alcohol-induced blackouts. Regarding behavioral factors, $59.8 \%$ of respondents participated in a pre-party or drinking games before an alcohol-induced blackouts, $54.7 \%$ did not eat correctly, and $79.5 \%$ reported having done something during the alcohol-induced blackouts that they regretted later. Conclusion. There is a high prevalence of alcohol-induced blackouts among medical students, which is associated with excessive alcohol consumption, having participated in preparties or games with drinks, not having eaten properly and regrets.

Keywords. Alcohol Drinking in College; Alcohol-Related Disorders; Risk Factors; Amnesia, Anterograde; Students, Medical

\section{Resumen}

Objetivo. Conocer la prevalencia y los factores asociados a la amnesia inducida por alcohol (AIA) en estudiantes de medicina de la Universidade do Extremo Sul Catarinense. Método. Investigación observacional transversal con enfoque cuantitativo. Realizado a través de un cuestionario online, elaborado por los propios autores, que contiene preguntas seleccionadas del Alcohol Use Disorder Identification Test (AUDIT). El cuestionario evaluó a la población académica $(n=268)$ del curso de medicina de la Universidade do Extremo Sul Catarinense en cuanto a su perfil, consumo de alcohol y AIA. Resultados: Se observó que de los estudiantes que beben de 2 a 4 veces al mes, el 48,9\% tenía AIA, mientras que de los que beben 4 o más veces a la semana, el 75\% tenía AIA. Al analizar por dosis consumidas por ocasión, de los que beben de 3 a 4 dosis el 47,6\% tenía AIA y de los que beben 10 o más dosis el $88 \%$ tenía AIA. Al analizar la frecuencia de binge drink, el 45,4\% de los que beben menos de uma vez al mes tenían AIA y el $67 \%$ semanalmente tenían AIA. No se encontró relación entre sexo, perfil de vivenda, semestre de graduación y AIA. En cuanto a los factores de comportamiento, el 59,8\% de los encuestados participó en alguna pre-fiesta o juego con bebidas antes del AIA, el 54,7\% no comió correctamente y el 79,5\% informó haber hecho algo durante el AIA de lo que luego se arrepintió. Conclusión: Existe una alta prevalencia de AIA entre los estudiantes de medicina, la cual se asocia con el consumo excesivo de alcohol, haber participado en prefiestas o juegos con bebidas, no comer adecuadamente y arrepentimientos.

Palabras clave. Consumo de Alcohol en la Universidad; Trastornos Relacionados con Alcohol; Factores de Riesgo; Amnesia Anterógrada; Estudiantes de Medicina

Trabalho realizado na Universidade do Extremo Sul Catarinense (UNESC), Criciúma-SC, Brasil.

Conflito de interesse: não

Recebido em: 29/07/2021

Aceito em: 06/12/2021

Endereço para correspondência: Eduardo P Rico. Universidade do Extremo Sul Catarinense (UNESC). Av. Universitária 1105. Bairro Universitário. CEP 88806-000. Criciúma-SC, Brasil. E-mail: eduardorico@unesc.net

\section{INTRODUÇÃO}

O consumo de álcool entre os jovens, em especial entre os estudantes universitários, é uma crescente preocupação. De acordo com um estudo do governo brasileiro, 86,2\% dos universitários referiram uso do álcool em algum momento da vida $^{1}$. Os estudantes de medicina estão presentes nestas estatísticas, entre 66,3\% e 97,3\% desses consomem álcool, 
mesmo sabendo os malefícios que seu uso pode causar ${ }^{2}$. Logo, os universitários são considerados uma população de risco para uso de álcool e suas consequências ${ }^{3}$.

Uma das consequências que o consumo excessivo e acelerado de álcool pode causar, principalmente quando a concentração de álcool no sangue (CAS) se eleva de forma rápida e atinge altos picos, é a Amnésia Induzida por Álcool $(A I A)^{4-6}$. Essa característica de consumo é frequente entre os universitários, por isso essa população acaba apresentando um maior risco de sofrer a AIA. Estudos sugerem que cerca de $50 \%$ ou mais dos universitários já apresentaram um episódio de AIA pelo menos uma vez na vida7-11.

A AIA pode ser definida como um período de amnésia anterógrada em que os indivíduos são incapazes de recordar elementos críticos de eventos, ou até mesmo eventos completos, que ocorreram enquanto estavam embriagados $^{12}$. Sugere-se que seja causada pelo impacto agudo do álcool no hipocampo, resultando em falha na transferência de informações da memória de curto prazo para a memória de longo prazo ${ }^{12}$. Assim, durante a AIA, a memória de curto prazo permanece intacta e o indivíduo está consciente. O sujeito é capaz de responder ao seu ambiente, e apresentar uma variedade de comportamentos, incluindo comportamentos de risco, tais como dirigir ou ter relação sexual desprotegida4. Entretanto, as informações sobre esses comportamentos não são transferidas para a memória de longo prazo, o que leva a déficits e perda de memória desses eventos ${ }^{4}$. 
Alguns fatores podem indicar um maior risco para o desenvolvimento da AIA, os mais comuns destes são: ser caucasiano, ter idade precoce de início do consumo de álcool, ter antecedentes familiares relacionados ao uso problemático de álcool, baixo peso corporal, uso de drogas múltiplas, maior frequência de binge drink (consumo de 5 ou mais doses para homens ou 4 ou mais doses para mulheres em uma ocasião) ${ }^{1}$, tabagismo, morar com outros estudantes, ingerir bebidas alcoólicas sem se alimentar, e rapidamente por um curto período de tempo, em especial durante jogos com bebidas ${ }^{6,10,13-15}$. Além disso, apenas $50 \%$ dos indivíduos que consomem álcool apresentam a AIA, sugerindo suscetibilidade genética como outro fator de risco associado ${ }^{10,16}$.

A ocorrência de uma AIA é preditiva para experimentar outros problemas relacionados ao álcool, como ressaca e overdose ${ }^{13}$. Porém, suas consequências vão além das relacionadas ao episódio de bebida para incluir sintomas psiquiátricos e anormalidades neurobiológicas ${ }^{4}$. Ademais, como a AIA está associada a comportamentos de risco, isso pode culminar em visitas a emergência, o que consequentemente eleva os custos em saúde ${ }^{11}$.

O melhor entendimento da AIA e de seus fatores associados pode ajudar a informar o quanto essa condição afeta a qualidade da saúde do universitário e o quanto impacta a saúde pública. Também pode colaborar no desenvolvimento de estratégias preventivas que possam reduzir estas consequências. Não foram encontradas 
evidências nacionais na literatura acerca do tema. Portanto, o objetivo desta pesquisa foi conhecer a prevalência e fatores associados à AIA em acadêmicos de medicina da Universidade do Extremo Sul Catarinense (UNESC).

\section{MÉTODO}

\section{Amostra}

O estudo recebeu aprovação do Comitê de Ética em Pesquisa e Humanos da UNESC, conforme CAAE: 35502720.3.0000.0119. Para participar da pesquisa, os voluntários concordaram com Termo de Consentimento Livre e Esclarecido (TCLE).

A pesquisa possuiu caráter observacional transversal, com coleta de dados primários e abordagem quantitativa. Foi realizada de forma online, entre 15 de dezembro a 31 de dezembro de 2020, através de um questionário eletrônico enviado para o e-mail de todos os acadêmicos matriculados no curso de medicina da UNESC no segundo semestre de 2020, que eram no total 807 alunos.

O tamanho mínimo da amostra, ou seja, de respondentes, foi calculado pela fórmula proposta por Medronho ${ }^{17}$, que resultou em 268 acadêmicos. O processo de coleta desse dado foi por amostragem estratificada, em que os estratos são os doze semestres do curso de medicina. Portanto, o número mínimo da amostra foi distribuído proporcionalmente entre os doze semestres, que resultou, em ordem crescente de semestres, em: 33, 33, 33, 20, 20, $20,19,19,19,17,18,17$ alunos participantes em cada 
semestre. De acordo com o cálculo estabelecido, foi utilizada neste estudo a totalidade de 268 acadêmicos participantes.

No estudo, foram incluídos os acadêmicos com idade igual ou superior a 18 anos, e excluídos aqueles que não concordaram com o TCLE e/ou que responderam ao questionário de forma incompleta.

\section{Procedimento}

Os dados foram coletados a partir de um questionário elaborado pelos autores, contendo perguntas selecionadas do Alcohol Use Disorder Identification Test (AUDIT), proposto pela Organização Mundial da Saúde (OMS) ${ }^{18}$ como instrumento de rastreamento do uso problemático de álcool. O questionário foi desenvolvido e disponibilizado por meio da plataforma Online Pesquisa (Copyright (C) 2007 - 2021 enuvo GmbH, Seefeldstrasse 25, 8008 Zurich, Switzerland), e enviado por e-mail através de um link para seu preenchimento online, para os 807 acadêmicos de medicina da UNESC.

No início do questionário, os pesquisadores apresentaram o TCLE e os objetivos da pesquisa, pedindo aos interessados que assinalassem a opção em que concordam com o TCLE. Assim, o participante era direcionado a primeira parte do questionário, desenvolvida para a caracterização do perfil do entrevistado, que continha informações relacionadas ao: sexo, idade, cor da pele, estado civil, escolaridade, moradia e semestre da graduação. 
A segunda parte do questionário foi composta por informações relacionadas ao consumo de álcool, a AIA e seus fatores associados. Possuía perguntas do AUDIT, como: frequência do uso de álcool; doses consumidas por ocasião; quantidade de vezes que foi incapaz de lembrar algo devido a bebida nos últimos 12 meses (AIA); e se já causou ferimentos ou prejuízos após ter bebido. Além de outras perguntas elaboradas pelos autores, que incluem: idade da primeira experimentação alcoólica; frequência de binge drink; se alguma vez foi incapaz de lembrar o que aconteceu devido a bebida (AIA) nos últimos 12 meses; se usou outra substância psicoativa junto ao episódio de AIA; se participou de pré-festas ou jogos de bebida antes de apresentar a AIA; se já se arrependeu de algo que não lembra (AIA); se teve alimentação adequada antes ou durante a AIA; e por fim, se a pandemia do COVID-19 alterou seus hábitos de ingestão alcoólica.

Os participantes que responderam "não" à questão "Alguma vez, ao longo dos últimos 12 meses, você foi incapaz de lembrar o que aconteceu devido a bebida (AIA)?", foram automaticamente direcionados ao final do questionário, pois não precisavam responder as outras perguntas relacionadas aos fatores associados a AIA, visto que não sofreram com uma recentemente.

\section{Análise Estatística}

Os testes estatísticos foram realizados com nível de significância $a=0,05$ e, portanto, confiança de 95\%. As 
variáveis quantitativas apresentaram dados paramétricos com distribuição normal, sendo, portanto, expressas como média e desvio padrão. Foram avaliadas quanto à normalidade por meio da aplicação do teste de Shapiro-Wilk e Kolmogorov-Smirnov, e comparadas suas médias por meio da aplicação do teste $\mathrm{H}$ de Kruskal-Wallis. A homogeneidade das variâncias foi avaliada por meio da aplicação do teste de Levene. As variáveis qualitativas foram expressas por meio de frequência e porcentagem. Foi realizada a investigação da existência da associação entre essas variáveis por meio da aplicação dos testes Qui-quadrado de Pearson e Razão de Verossimilhança, seguidos de análise de resíduo quando observada significância estatística.

\section{RESULTADOS}

Os resultados presentes na Tabela 1 referem-se à caracterização do perfil dos 268 acadêmicos participantes. Diante disto, foi observado que a média de idade foi de 22,5 anos, sendo a maior parte composta pelo sexo feminino $(67,5 \%)$, da raça branca $(95,9 \%)$, solteiro $(95,9 \%)$, que ainda moram com os pais ou alguém da família $(63,4 \%)$ e possuem o ensino superior incompleto ( $81 \%)$, ou seja, estão na sua primeira graduação. Em relação ao semestre da graduação, sua distribuição foi proporcional, como o cálculo da amostra requisitou. 
Tabela 1. Caracterização da Amostra.

\begin{tabular}{|c|c|}
\hline & $\begin{array}{c}\text { Média } \pm D P, n(\%) \\
n=268\end{array}$ \\
\hline Idade (anos) & $22,50 \pm 3,11$ \\
\hline \multicolumn{2}{|l|}{ Sexo } \\
\hline Feminino & $181(67,5)$ \\
\hline Masculino & $87(32,5)$ \\
\hline \multicolumn{2}{|l|}{ Raça } \\
\hline Branca & $257(95,9)$ \\
\hline Preta & $4(1,5)$ \\
\hline Outra & $7(2,6)$ \\
\hline \multicolumn{2}{|l|}{ Estado civil } \\
\hline Solteiro (a) & $257(95,9)$ \\
\hline Casado (a) ou união estável & $11(4,1)$ \\
\hline \multicolumn{2}{|l|}{ Moradia } \\
\hline Com outro (s) estudante (s) & $34(12,7)$ \\
\hline Com os pais ou alguém da família & $170(63,4)$ \\
\hline Sozinho & $58(21,6)$ \\
\hline Outra & $6(2,2)$ \\
\hline \multicolumn{2}{|l|}{ Escolaridade } \\
\hline Ensino superior incompleto & $217(81,0)$ \\
\hline Ensino superior completo & $51(19,0)$ \\
\hline \multicolumn{2}{|l|}{ Fase } \\
\hline $1^{\mathrm{a}}$ & $33(12,3)$ \\
\hline $2^{a}$ & $33(12,3)$ \\
\hline $3^{a}$ & $33(12,3)$ \\
\hline $4^{a}$ & $20(7,5)$ \\
\hline $5 a$ & $20(7,5)$ \\
\hline $6^{a}$ & $20(7,5)$ \\
\hline $7^{a}$ & $19(7,1)$ \\
\hline $8^{a}$ & $19(7,1)$ \\
\hline $9 a$ & $19(7,1)$ \\
\hline $10^{a}$ & $17(6,3)$ \\
\hline $11^{a}$ & $18(6,7)$ \\
\hline $12^{\mathrm{a}}$ & $17(6,3)$ \\
\hline
\end{tabular}

Com relação ao consumo de álcool entre os acadêmicos de medicina, os resultados se encontram na Tabela 2, e apontaram uma média de idade da primeira experimentação alcoólica em torno dos 15 anos. Foi constatado que a maioria dos acadêmicos relatou consumir álcool em uma frequência maior ou igual a 2 a 4 vezes ao mês $(72,1 \%)$, e nas ocasiões em que o álcool é consumido, a maioria ingere 3 a mais doses $(62,4 \%)$. 
Tabela 2. Consumo de álcool entre os acadêmicos de medicina.

\begin{tabular}{|c|c|}
\hline & $\begin{array}{l}\text { Média } \pm D P, \text { n (\%) } \\
n=268\end{array}$ \\
\hline Idade que experimentou álcool pela primeira vez (anos) & $14,94 \pm 5,53$ \\
\hline \multicolumn{2}{|l|}{ Com que frequência você consome bebidas alcoólicas? } \\
\hline Nunca & $13(4,9)$ \\
\hline Mensalmente ou menos & $62(23,1)$ \\
\hline De 2 a 4 vezes por mês & $143(53,4)$ \\
\hline De 2 a 4 vezes por semana & $46(17,2)$ \\
\hline 4 ou mais vezes por semana & $4(1,5)$ \\
\hline \multicolumn{2}{|c|}{$\begin{array}{l}\text { Nas ocasiões em que ingere bebidas alcoólicas, quantas doses em } \\
\text { média são consumidas? }\end{array}$} \\
\hline Não ingiro & $14(5,2)$ \\
\hline De 1 a 2 & $87(32,5)$ \\
\hline De 3 a 4 & $103(38,4)$ \\
\hline De 5 a 6 & $39(14,6)$ \\
\hline De 7 a 9 & $16(6,0)$ \\
\hline 10 ou mais & $9(3,4)$ \\
\hline \multicolumn{2}{|c|}{$\begin{array}{l}\text { Com que frequência você bebe } 5 \text { ou mais doses (homem), ou } 4 \\
\text { ou mais doses (mulher) de uma vez? }\end{array}$} \\
\hline Nunca & $46(17,2)$ \\
\hline Menos do que uma vez por mês & $141(52,6)$ \\
\hline Mensalmente & $56(20,9)$ \\
\hline Semanalmente & $24(9,0)$ \\
\hline \multicolumn{2}{|c|}{$\begin{array}{l}\text { Você considera que a pandemia do covid-19 afetou seus hábitos } \\
\text { de ingestão alcoólica? }\end{array}$} \\
\hline Sim, para menos & $127(47,4)$ \\
\hline Sim, para mais & $72(26,9)$ \\
\hline Não & $69(25,7)$ \\
\hline
\end{tabular}

Também foi encontrada uma elevada frequência de binge drink, com os estudantes relatando consumirem nesse padrão mensalmente e semanalmente (29,9\%). Porém, considerando que aproximadamente metade dos entrevistados relatou ter reduzido seu consumo alcoólico durante a pandemia $(47,4 \%)$, todos os resultados sobre consumo de álcool e a AIA podem ter sido atenuados.

Em relação ao episódio de AIA, os resultados se encontram na Tabela 3, e demostraram que quase a metade dos estudantes de medicina relatou ter AIA nos últimos 12 
meses $(43,7 \%)$. Entre esses, grande parte relatou que a AIA ocorreu em uma frequência menor do que mensalmente $(88,1 \%)$.

Tabela 3. Prevalência de AIA.

\begin{tabular}{|c|c|}
\hline & $\begin{array}{l}n(\%) \\
n=268\end{array}$ \\
\hline \multicolumn{2}{|c|}{$\begin{array}{l}\text { Alguma vez, nos últimos } 12 \text { meses, você foi incapaz de lembrar o que } \\
\text { aconteceu devido à bebida (AIA)? }\end{array}$} \\
\hline Não & $151(56,3)$ \\
\hline Sim & $117(43,7)$ \\
\hline \multicolumn{2}{|c|}{$\begin{array}{l}\text { Quantas vezes, ao longo dos últimos } 12 \text { meses, você foi incapaz de } \\
\text { lembrar o que aconteceu devido à bebida (AIA)? }\end{array}$} \\
\hline Menos do que uma vez ao mês & $103(88,1)$ \\
\hline Mensalmente & $12(10,3)$ \\
\hline Semanalmente & $1(0,8)$ \\
\hline Todos ou quase todos os dias & $1(0,8)$ \\
\hline Não informado & 151 \\
\hline
\end{tabular}

Quanto à associação de consumo de álcool e a presença de AIA, os resultados se encontram na Tabela 4, e mostraram que $84,6 \%$ dos estudantes que relataram ter tido AIA nos últimos 12 meses consomem álcool em uma frequência igual ou maior que 2 a 4 vezes ao mês $(p<0,001)$, $76 \%$ inferem consumir igual ou mais de 3 a 4 doses por ocasião $(p<0,001)$, e 43,6\% relatam consumir em binge drink em uma frequência igual ou maior que mensalmente $(p<0,001)$. Ao comparar às faixas de frequência de consumo e ter apresentado AIA, foi observado que quanto maior a frequência de consumo de álcool, maior é a chance de o indivíduo ter apresentado AIA (35,3\% dos que bebem mensalmente ou menos $\times 75 \%$ dos que bebem 4 ou mais vezes por semana). 
Tabela 4. Consumo de álcool x presença de AIA

\begin{tabular}{|c|c|c|c|c|c|}
\hline & \multicolumn{2}{|c|}{$\begin{array}{l}\text { Alguma vez, nos últimos } 12 \\
\text { meses, você teve um episódio } \\
\text { de AIA? }\end{array}$} & \multirow[t]{3}{*}{ Valor $-\mathbf{p}^{+}$} & \multicolumn{2}{|c|}{ Comparação entre as frequências e ter tido AIA } \\
\hline \multirow[b]{3}{*}{$\begin{array}{l}\text { Com que } \\
\text { frequência você } \\
\text { toma bebidas } \\
\text { alcoólicas? }\end{array}$} & Não & Sim & & & \\
\hline & $\mathbf{n}=\mathbf{1 5 1}$ & $n=117$ & & & \\
\hline & & & & Não - n (\%) & Sim - n (\%) \\
\hline Nunca & $13(8,6)^{b}$ & $0(0,0)$ & $<0,001$ & $13(100)$ & $0(0)$ \\
\hline $\begin{array}{l}\text { Mensalmente ou } \\
\text { menos }\end{array}$ & $44(29,1)^{b}$ & $18(15,4)$ & & $44(64,7)$ & $18(35,3)$ \\
\hline $\begin{array}{l}\text { De } 2 \text { a } 4 \text { vezes } \\
\text { por mês }\end{array}$ & $73(48,3)$ & $70(59,8)$ & & $73(51,1)$ & $70(48,9)$ \\
\hline $\begin{array}{l}\text { De } 2 \text { a } 4 \text { vezes } \\
\text { por semana }\end{array}$ & $20(13,2)$ & $26(22,2)$ & & $20(43,8)$ & $26(56,2)$ \\
\hline $\begin{array}{l}4 \text { ou mais vezes } \\
\text { por semana }\end{array}$ & $1(0,7)$ & $3(2,6)$ & & $1(25)$ & $3(75)$ \\
\hline
\end{tabular}

Comparação entre a quantidade de doses ingeridas e ter tido AIA

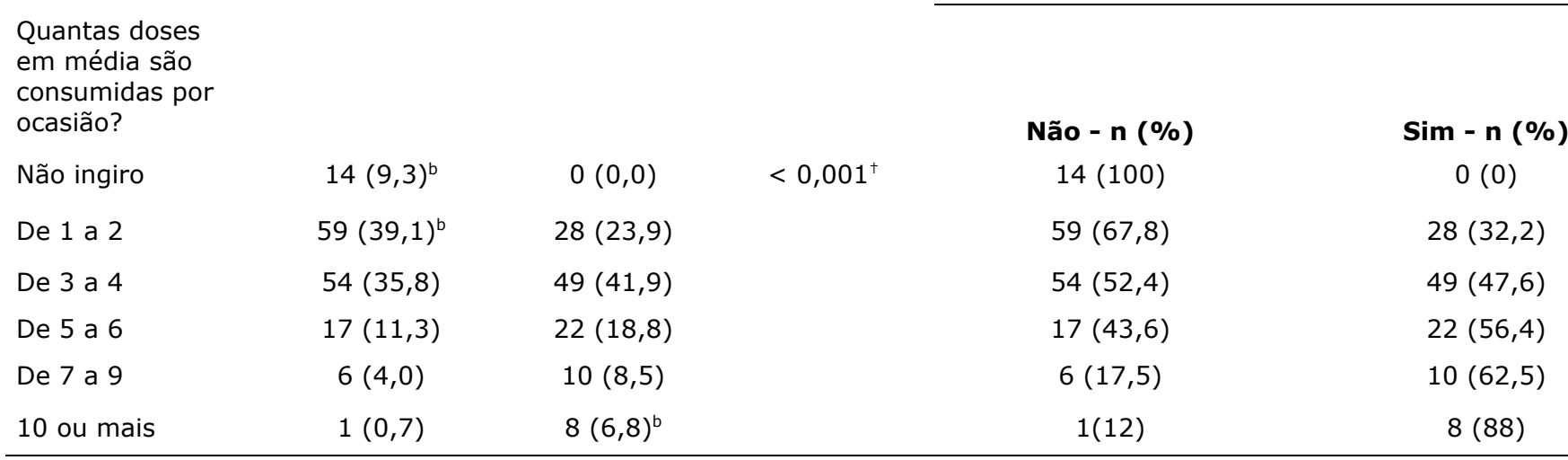

Comparação entre a frequência de binge drink e ter

Com que

frequência você

toma 5 ou mais

doses (homem),

ou 4 ou mais

doses (mulher) de uma vez?

Nunca

$44(29,1)^{b}$

$2(1,7)$

$<0,001$

Não - n (\%)

Sim - n (\%)

tido AIA

Menos do que

uma vez por mês

$77(51,0) \quad 64(54,7)$

$44(95,7)$

$2(4,3)$

Mensalmente

$21(13,9) \quad 35(29,9)^{\mathrm{b}}$

$77(54,6)$

$64(45,4)$

Semanalmente

$8(5,3)$

$16(13,7)^{\mathrm{b}}$

$21(37,5)$

$35(62,5)$

8 (33)

16(67)

Todos ou quase todos os dias

$1(0,7) \quad 0(0,0)$

$1(100)$

$0(0)$

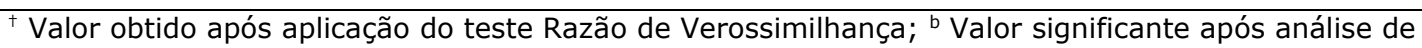
resíduo; 
O mesmo fato acontece ao analisar por faixas de doses consumidas por ocasião, quanto maior a quantidade de doses consumidas, maior é a probabilidade de o indivíduo ter apresentado AIA (32,2\% dos que ingerem de 1 a 2 doses $x$ $88 \%$ dos que ingerem 10 ou mais doses). Por fim, ao analisar as faixas de frequência de beber em binge drink, foi observado também que quanto a maior frequência de binge drink, maior a chance de ter apresentado AIA $(45,4 \%$ dos que bebem menos que mensalmente $x 67 \%$ dos que bebem semanalmente).

Em relação aos fatores comportamentais associados à AIA, os resultados se encontram na Tabela 5, e apontaram que a maioria dos participantes não usou outra substância psicoativa junto ao álcool quando apresentou a AIA (81,2\%), participou de alguma pré-festa ou jogos com bebidas antes da AIA (59,8\%), e que não se alimentou corretamente antes ou durante a AIA $(54,7 \%)$. Além disso, grande parte dos acadêmicos que apresentaram AIA relatou ter se arrependido de algo (79,5\%), e alguns chegaram a causar ferimentos ou prejuízos a ele mesmo ou a outra pessoa durante o episódio (17,9\%).

A respeito dos fatores sociodemográficos associados à AIA, foram analisadas as variáveis sobre moradia, sexo e semestre da graduação, listadas na Tabela 6. Os resultados obtidos não foram estatisticamente significativos $(p>0,05)$. Tal achado evidencia que aspectos como morar sozinho ou compartilhando residência com colegas, ser do sexo feminino 


\section{ou masculino, ou estar em qualquer semestre da graduação não tem correlação positiva com a AIA.}

Tabela 5. Fatores comportamentais associados a AIA.

\begin{tabular}{|c|c|}
\hline & $\begin{array}{l}n(\%) \\
n=268\end{array}$ \\
\hline \multicolumn{2}{|c|}{$\begin{array}{l}\text { Você usou outra substância psicoativa junto com o álcool quando sofreu o } \\
\text { episódio de amnésia? }\end{array}$} \\
\hline Não, apenas o álcool & $95(81,2)$ \\
\hline Sim, usei cigarro & $9(7,7)$ \\
\hline Sim, usei maconha & $4(3,4)$ \\
\hline Sim, usei outro tipo de droga & $6(5,1)$ \\
\hline Sim, usei 2 ou mais drogas junto & $3(2,6)$ \\
\hline Não informado & 151 \\
\hline \multicolumn{2}{|c|}{$\begin{array}{l}\text { Você participou de pré-festas (esquenta(o)) ou de jogos de beber antes de } \\
\text { sofrer o episódio de amnésia alcoólica? }\end{array}$} \\
\hline Sim & $70(59,8)$ \\
\hline Não & $47(40,2)$ \\
\hline Não informado & 151 \\
\hline \multicolumn{2}{|c|}{$\begin{array}{l}\text { Você se alimentou bem antes ou durante o evento que desencadeou do } \\
\text { episódio de amnésia? }\end{array}$} \\
\hline Não & $64(54,7)$ \\
\hline Não sei dizer & $29(24,8)$ \\
\hline Sim & $24(20,5)$ \\
\hline Não informado & 151 \\
\hline \multicolumn{2}{|c|}{$\begin{array}{l}\text { Você já fez algo que se arrependeu durante o episódio de amnésia } \\
\text { alcoólica? Ou seja, você fez algo que se arrependeu, mas que não lembra } \\
\text { (alguém te contou) ou lembra em partes. }\end{array}$} \\
\hline Sim & $93(79,5)$ \\
\hline Não & $24(20,5)$ \\
\hline Não informado & 151 \\
\hline \multicolumn{2}{|c|}{$\begin{array}{l}\text { Alguma vez na vida você já causou ferimentos ou prejuízos a você mesmo } \\
\text { ou a outra pessoa após ter bebido? }\end{array}$} \\
\hline Não & $82(70,1)$ \\
\hline Sim, mas não junto à amnesia induzida por álcool & $14(12,0)$ \\
\hline Sim, junto à amnesia induzida por álcool & $21(17,9)$ \\
\hline Não informado & 151 \\
\hline
\end{tabular}


Tabela 6. Fatores sociodemográficos associados a AIA.

\begin{tabular}{|c|c|c|c|}
\hline & \multicolumn{2}{|c|}{$\begin{array}{c}\text { Alguma vez, nos últimos } 12 \text { meses, você } \\
\text { teve um episódio de AIA? }\end{array}$} & \multirow{2}{*}{$\begin{array}{l}\text { Valor - } \\
\quad \text { p }\end{array}$} \\
\hline & $\begin{array}{c}\text { Não } \\
n=151\end{array}$ & $\begin{array}{c}\operatorname{Sim} \\
n=117 \\
\end{array}$ & \\
\hline \multicolumn{4}{|l|}{ Moradia } \\
\hline Com outro (s) estudante (s) & $12(7,9)$ & $22(18,8)$ & $0,065^{+}$ \\
\hline Com os pais ou alguém da família & $101(66,9)$ & $69(59,0)$ & \\
\hline Sozinho & $35(23,2)$ & $23(19,7)$ & \\
\hline Outra & $3(2,0)$ & $3(2,6)$ & \\
\hline \multicolumn{4}{|l|}{ Sexo } \\
\hline Masculino & $51(33,8)$ & $36(30,8)$ & $0,602^{\ddagger}$ \\
\hline Feminino & $100(66,2)$ & $81(69,2)$ & \\
\hline \multicolumn{4}{|l|}{ Fase } \\
\hline 1 a 3 & $58(58,6)$ & $41(41,4)$ & $0,304^{\ddagger}$ \\
\hline 4 a 6 & $30(50,0)$ & $30(50,0)$ & \\
\hline 7 a 9 & $29(50,9)$ & $28(49,1)$ & \\
\hline 10 a 12 & $34(65,4)$ & $18(34,6)$ & \\
\hline
\end{tabular}

${ }^{\ddagger}$ Valor obtido após aplicação do teste Qui-Quadrado de Pearson; ${ }^{\dagger}$ Valor obtido após aplicação do teste Razão de Verossimilhança.

\section{DISCUSSÃO}

Os resultados deste estudo apontam uma alta prevalência de AIA entre os acadêmicos de medicina nos últimos 12 meses. Esse achado corrobora com outros estudos realizados em universitários em geral, que demonstraram que $50 \%$ ou mais dos universitários já relataram um episódio de AIA pelo menos uma vez na vida711. Isso acontece, pois os estudantes acreditam que a universidade seja um período favorável para o consumo excessivo de álcool, já que estão relativamente livres das responsabilidades dos adultos, longe de figuras de autoridade, toleram bem as consequências da intoxicação alcoólica, e estão em ambientes legais para beber, onde podem realizar práticas de consumo acelerado de álcool ${ }^{6,19}$. Além disso, eles distinguem a bebida como algo que 
proporciona benefícios sociais, como vínculo, socialização, e desinibição, favorecendo mais seu uso ${ }^{20}$. Portanto, não é surpreendente que a AIA seja prevalente em universitários ${ }^{6}$.

Particularmente, os acadêmicos de medicina estão expostos a vários fatores estressantes durante a graduação, como grande pressão de aprendizado, processar grande quantidade de novas informações, falta de tempo para atividades sociais e ter contato com doenças graves e com a morte $^{21}$. Por consequência, os alunos podem abusar de substâncias, como uma tentativa de escapar e aliviar sua realidade. O álcool é a substância mais consumida pelos estudantes brasileiros de medicina, sendo a prevalência do consumo entre $66,3 \%$ e $97,3 \%^{2}$. O presente estudo apresenta estatísticas semelhantes, ao mostrar que $96,1 \%$ da amostra consumiu álcool nos últimos 12 meses.

$\mathrm{Na}$ relação à associação de consumo de álcool e a presença de AIA, os resultados demonstram que quanto maiores forem os excessos de consumo de álcool, seja na quantidade de doses, frequência de consumo alcoólico, ou na frequência de binge drink, há maiores probabilidades do entrevistado já ter tido um episódio de AIA naquele ano. Esse fato acontece visto que à medida que a quantidade de álcool consumida aumenta, aumenta também a magnitude das deficiências de memória ${ }^{12}$. Assim, pessoas com o padrão de consumo alcoólico mais alto, como o binge drink, apresentam mais chances de sofrer um episódio de AIA ${ }^{22}$.

A ligação entre binge drink e AIA é bem estabelecida na literatura, mas também há outras causas diretas, como um 
alto pico e uma rápida elevação da $\mathrm{CAS}^{5,10}$. Essa situação ocorre principalmente quando grandes quantidades de álcool são ingeridas em ritmo acelerado em períodos curtos de tempo, prática comum durante pré-festas ou jogos de bebida $^{6,14}$ (consumo de álcool antes de participar de um evento planejado, por exemplo, festa, em que mais álcool pode ou não ser consumido) ${ }^{23}$. Além disso, engolir bebidas e ingerir álcool de estômago vazio, também podem elevar rapidamente a CAS, aumentando a probabilidade da AIA $^{5,15}$. Este estudo reforça o que é descrito na literatura, visto que a maioria dos entrevistados participou de pré-festas ou jogos com bebidas e se alimentou de forma inadequada antes ou durante a AIA.

Diferente da literatura, não foi encontrada relação entre o uso simultâneo de outras drogas ao episódio de AIA, visto que a maioria da amostra experimentou a AIA apenas usando álcool. É difícil determinar se o abuso simultâneo de substâncias influencia na AIA, especialmente pelos indivíduos que apresentaram a AIA não serem capazes de recordar completamente dos eventos, como por exemplo, o uso de outras drogas durante a AIA. Porém, outros estudos já associaram um maior risco de AIA ao tabagismo, ao uso de maconha e a múltiplas drogas ${ }^{13,24}$.

Outra questão abordada são as consequências negativas futuras que a AIA pode causar. Os resultados mostraram que a grande parte dos participantes se arrependeu de algo que fez durante a AIA. Um dos motivos disso ocorrer é justamente pela distorção das memórias dos 
eventos. Os indivíduos na AIA podem ter comportamentos não planejados, divergentes dos seus valores quando sóbrios, além de estarem menos conscientes das pessoas ao seu redor, sendo mais provável que façam algo que depois se arrependam ${ }^{4,7,8}$. Também apresentar uma AIA é fator preditor significativo de ter outros problemas relacionados ao álcool, como ressaca, faltar ou se atrasar para um compromisso, ter problemas com a polícia, se machucar e necessitar de serviço médico por overdose ${ }^{13}$. Todas essas situações contribuem para reações emocionais negativas, que podem perdurar ao longo do tempo.

Na questão de saúde pública, a ocorrência generalizada de AIA entre estudantes universitários representa uma séria preocupação. Foi mostrado que uma a cada oito visitas ao departamento de emergência por lesões relacionadas ao álcool envolveram um episódio de AIA, gerando custos aos sistemas de saúde ${ }^{11}$. Além disso, alunos que apresentam episódios de AIA frequentes têm quase três vezes mais probabilidade de sofrer uma lesão relacionada ao álcool durante os próximos dois anos do que aqueles que não tiveram $\mathrm{AIA}^{22}$. Pesquisas recentes também apontaram que 0 consumo excessivo de álcool com uma história positiva de AIA, leva a transtornos psiquiátricos, como ideação e tentativas de suicídio ${ }^{25}$, e depressão ${ }^{26}$. Soma-se a isso, a associação entre AIA e outros comportamentos de risco à saúde, como o uso de álcool ou drogas antes de fazer sexo e ser vítima de agressão sexual, ressaltando a seriedade desse problema na saúde pública ${ }^{24}$. 
Outro fato importante é que a maturação neurológica de um indivíduo só se completa ao fim da adolescência e início da vida adulta, durante esse tempo o uso intenso de álcool por afetar o desenvolvimento de regiões do cérebro e interferir no funcionamento cognitivo ${ }^{27}$. Já foi observado, que acadêmicos de medicina têm seu desempenho afetado após um dia de bebedeira e que provavelmente algum dano é causado na vida acadêmica desse estudante ${ }^{28}$. Ademais, experimentar três ou mais episódios de AIA foi associado a tirar notas mais baixas ${ }^{10}$. A literatura defende que as universidades incluam no currículo do curso de medicina programas de educação e prevenção sobre o uso de álcool e demais drogas, pois se trata de um perfil de pessoas que serão as responsáveis por promover a saúde futuramente ${ }^{28}$.

O presente estudo tem a função de auxiliar nessa jornada, gerando uma maior compreensão sobre a AIA e o padrão de consumo alcoólico entre os acadêmicos de medicina. Uma análise específica de dados sobre universitários facilita o desenvolvimento de ações intervencionistas mais diretas aos fatores predisponentes dessa população, melhorando a qualidade dos futuros médicos e consequentemente 0 atendimento que $a$ sociedade irá receber.

\section{Limitações}

O estudo traz como limitações o fato do questionário ser um auto relato, e, portanto, os participantes podem responder de um modo não fidedigno a sua realidade, 
principalmente atenuando seus comportamentos alcoólicos e uso de substâncias. O viés da memória também permanece uma possibilidade, pois uma vez acometida poderia alterar as respostas fornecidas pelos estudantes. Ademais, apesar de o estudo ter tido a oportunidade de avaliar as mudanças de hábitos alcoólicos durante a pandemia do COVID-19, esse cenário pode ter atenuado os resultados sobre a AIA, visto que esta é associada aos excessos de consumos alcoólicos, situações que ocorrem mais em aglomerações (pré-festas e festas), eventos proibidos durante o período da pesquisa.

\section{CONCLUSÃO}

Existe uma alta prevalência de AIA nos estudantes de medicina, sendo esta associada ao consumo de álcool em excesso, ter participado de pré-festas ou jogos com bebidas, e não ter se alimentado corretamente. Não foi encontrada relação entre AIA, perfil de moradia e sexo. Além disso, muitos estudantes referem se arrepender de algo feito durante a AIA, podendo gerar reações emocionais negativas. As universidades devem discutir e orientar seus estudantes quanto ao tema. Assim, os acadêmicos serão capazes de reconhecer os riscos e os padrões envolvidos na AIA e se tornarem capazes de evitá-los, beneficiando não apenas a qualidade dos futuros médicos e diminuição de gastos públicos, mas sim, a sociedade em geral. 


\section{AGRADECIMENTOS}

A todos os estudantes que participaram voluntariamente deste estudo, ao suporte técnico dos monitores e a plataforma Online Pesquisa pelo auxílio na coleta e armazenamento de dados.

\section{REFERÊNCIAS}

1.Brasil. Presidência da República. Secretaria Nacional de Políticas sobre Drogas. Andrade AG, Duarte PCAV, Oliveira LG (org.) I Levantamento Nacional sobre o Uso de Álcool, Tabaco e Outras Drogas entre Universitários das 27 Capitais Brasileiras / Secretaria Nacional de Políticas sobre Drogas; GREA/IPQ-HC/FMUSP. Brasília: SENAD, 2010. $284 \mathrm{p}$.

http://www.mds.gov.br/webarquivos/arquivo/cuidados prevencao dr ogas/obid/publicacoes/Livros/I\%20Levantamento\%20Nacional\%20Un iversit\%C3\%A1rios\%20-\%202010.pdf

2.Candido FJ, Souza R, Stumpf MA, Fernandes LG, Veiga R, Santin M, et al. The use of drugs and medical students: a literature review. Rev Assoc Med Bras 2018;64:462-8. https://doi.org/10.1590/1806$\underline{9282.64 .05 .462}$

3. Hingson RW, Zha W, Weitzman ER. Magnitude of and Trends in Alcohol-Related Mortality and Morbidity Among U.S. College Students Ages 18-24, 1998-2005. J Stud Alcohol Drugs 2009;(suppl16):12-20. https://doi.org/10.15288/jsads.2009.s16.12

4. Wetherill RR, Fromme K. Alcohol-Induced Blackouts: A Review of Recent Clinical Research with Practical Implications and Recommendations for Future Studies. Alcohol Clin Exp Res 2016;40:922-35. https://doi.org/10.1111/acer.13051

5.Perry PJ, Argo TR, Barnett MJ, Liesveld JL, Liskow B, Hernan JM, et al. The Association of Alcohol-Induced Blackouts and Grayouts to Blood Alcohol Concentrations. J Forensic Sci 2006;51:896-9. https://doi.org/10.1111/j.1556-4029.2006.00161.x

6.LaBrie JW, Hummer J, Kenney S, Lac A, Pedersen E. Identifying Factors That Increase the Likelihood for Alcohol-Induced Blackouts in the Prepartying Context. Subst Use Misuse 2011;46:992-1002. https://doi.org/10.3109/10826084.2010.542229

7. Barnett NP, Clerkin EM, Wood M, Monti PM, O'Leary T, Corriveau D, et al. Description and Predictors of Positive and Negative AlcoholRelated Consequences in the First Year of College. J Stud Alcohol Drugs 2014;75:103-14. https://doi.org/10.15288/jsad.2014.75.103

8. Wilhite ER, Fromme K. Alcohol-Induced Blackouts and Other Negative Outcomes During the Transition Out of College. J Stud Alcohol Drugs 2015;76:516-24. https://doi.org/10.15288/jsad.2015.76.516 
9.Marino EN, Fromme K. Alcohol-induced blackouts and maternal family history of problematic alcohol use. Addict Behav 2015;45:2016. https://doi.org/10.1016/j.addbeh.2015.01.043

10. White AM, Jamieson-Drake DW, Swartzwelder HS. Prevalence and Correlates of Alcohol-Induced Blackouts Among College Students: Results of an E-Mail Survey. J Am Coll Heal 2002;51:117-31. https://doi.org/10.1080/07448480209596339

11.Mundt MP, Zakletskaia LI. Prevention For College Students Who Suffer Alcohol-Induced Blackouts Could Deter High-Cost Emergency Department Visits. Health Aff 2012;31:863-70. https://doi.org/10.1377/hlthaff.2010.1140

12. White AM. What happened? Alcohol, memory blackouts, and the brain. Alcohol Res Health 2003;27:186-96. http://www.ncbi.nlm.nih.gov/pubmed/15303630

13. Hingson R, Zha W, Simons-Morton B, White A. Alcohol-Induced Blackouts as Predictors of Other Drinking Related Harms Among Emerging Young Adults. Alcohol Clin Exp Res 2016;40:776-84. https://doi.org/10.1111/acer. 13010

14.Ray AE, Stapleton JL, Turrisi R, Mun E-Y. Drinking game play among first-year college student drinkers: An event-specific analysis of the risk for alcohol use and problems. Am J Drug Alcohol Abuse 2014;40:353-8. https://doi.org/10.3109/00952990.2014.930151

15.Giles SM, Champion H, Sutfin EL, McCoy TP, Wagoner K. Calorie Restriction on Drinking Days: An Examination of Drinking Consequences Among College Students. J Am Coll Heal 2009;5:60310. https://doi.org/10.3200/JACH.57.6.603-610

16. Nelson EC, Heath AC, Bucholz KK, Madden PAF, Fu Q, Knopik V, et al. Genetic Epidemiology of Alcohol-Induced Blackouts. Arch Gen Psychiatry 2004;61:257-63.

https://doi.org/10.1001/archpsyc.61.3.257

17. Medronho RA. Epidemiologia. 2a. ed. São Paulo: Atheneu; 2009. $676 \mathrm{p}$.

18. Carneiro APL, Ronzani TM, Avallone DDM, Formigoni MLO de S. AUDIT (Alcohol Use Disorders Identification Test) \& AUDIT-C (endereço na Internet). Aberta: Portal de Formação a Distância. Sujeitos, contextos e drogas. (acessado em: 2021). Disponível em: https://edisciplinas.usp.br/pluginfile.php/4170599/mod resource/con tent/1/audit.pdf

19.Crawford LA, Novak KB, Jayasekare RR. Volunteerism, Alcohol Beliefs, and First-Year College Students' Drinking Behaviors: Implications for Prevention. J Prim Prev 2019;40:429-48. https://doi.org/10.1007/s10935-019-00558-z

20.Brown R, Murphy S. Alcohol and social connectedness for new residential university students: implications for alcohol harm reduction. J Furth High Educ 2020;44:216-30.

https://doi.org/10.1080/0309877X.2018.1527024

21.Lins L, Carvalho FM, Menezes MS, Porto-Silva L, Damasceno H. Health-related quality of life of students from a private medical school in Brazil. Int J Med Educ 2015;6:149-54. 
https://dx.doi.org/10.5116/ijme.563a.5dec

22.Mundt MP, Zakletskaia LI, Brown DD, Fleming MF. Alcohol-induced memory blackouts as an indicator of injury risk among college drinkers. Inj Prev 2012;18:44-9. http://dx.doi.org/10.1136/ip.2011.031724

23.Kenney SR, Hummer JF, LaBrie JW. An Examination of Prepartying and Drinking Game Playing During High School and Their Impact on Alcohol-Related Risk Upon Entrance into College. J Youth Adolesc 2010;39:999-1011. https://doi.org/10.1007/s10964-009-9473-1

24.Voloshyna DM, Bonar EE, Cunningham RM, Ilgen MA, Blow FC, Walton MA. Blackouts among male and female youth seeking emergency department care. Am J Drug Alcohol Abuse 2018;44:12939. https://doi.org/10.1080/00952990.2016.1265975

25.Bae H-C, Hong S, Jang S-I, Lee K-S, Park E-C. Patterns of Alcohol Consumption and Suicidal Behavior: Findings From the Fourth and Fifth Korea National Health and Nutritional Examination Survey (20072011). J Prev Med Public Heal 2015;48:142-50.

https://doi.org/10.3961/jpmph.14.027

26. Neupane SP, Bramness JG. Prevalence and correlates of major depression among Nepalese patients in treatment for alcohol-use disorders. Drug Alcohol Rev 2013;32:170-7.

https://doi.org/10.1111/j.1465-3362.2012.00487.x

27.Squeglia LM, Pulido C, Wetherill RR, Jacobus J, Brown GG, Tapert SF. Brain Response to Working Memory Over Three Years of Adolescence: Influence of Initiating Heavy Drinking. J Stud Alcohol Drugs 2012;73:749-60. https://doi.org/10.15288/jsad.2012.73.749

28.Carneiro EB, Braga RT, Silva LFD, Nogueira MC. Fatores associados a beber pesado episódico entre estudantes de medicina. Rev Bras Educ Med 2012;36:524-30. https://doi.org/10.1590/S0100$\underline{55022012000600011}$ 